\title{
Gap Solitons in a Spin-Orbit-Coupled Bose-Einstein Condensate
}

\author{
Yaroslav V. Kartashov, ${ }^{1,2}$ Vladimir V. Konotop, ${ }^{3}$ and Fatkhulla Kh. Abdullaev ${ }^{4}$ \\ ${ }^{1}$ ICFO-Institut de Ciencies Fotoniques and Universitat Politecnica de Catalunya, 08860 Castelldefels (Barcelona), Spain \\ ${ }^{2}$ Institute of Spectroscopy, Russian Academy of Sciences, Troitsk, Moscow Region 142190, Russia \\ ${ }^{3}$ Departamento de Física, Faculdade de Ciências and Centro de Física Teórica e Computacional Universidade de Lisboa, \\ Avenida Professor Gama Pinto 2, Lisboa 1649-003, Portugal \\ ${ }^{4}$ Instituto de Física Teórica, Universidade Estadual Paulista, 01140-070 Sao Paulo, Brasil
}

(Received 28 April 2013; published 7 August 2013)

\begin{abstract}
We report a diversity of stable gap solitons in a spin-orbit-coupled Bose-Einstein condensate subject to a spatially periodic Zeeman field. It is shown that the solitons can be classified by the main physical symmetries they obey, i.e., symmetries with respect to parity $(P)$, time $(T)$, and internal degree of freedom, i.e., spin $(C)$, inversions. The conventional gap and gap-stripe solitons are obtained in lattices with different parameters. It is shown that solitons of the same type but obeying different symmetries can exist in the same lattice at different spatial locations. $P T$ and $C P T$ symmetric solitons have antiferromagnetic structure and are characterized, respectively, by nonzero and zero total magnetizations.
\end{abstract}

DOI: 10.1103/PhysRevLett.111.060402

PACS numbers: 03.75.Lm, 03.75.Mn, 71.70.Ej

The progressively growing interest in the physics of mixtures of spinor Bose-Einstein condensates (BECs) and, in particular, in spin-orbit- (SO) coupled BECs $[1,2]$, is motivated on the one hand by their fundamental importance for the atomic physics, and, on the other hand, by the rich possibilities they offer for emulating synthetic electric and magnetic fields in solids [3]. The last property allows one using neutral atoms to simulate numerous condensed matter phenomena [4] in a tunable way, what would be impossible in direct condensed matter experiments. In other words, a spinor BEC is a promising candidate for implementation of a quantum simulator, which due to the technologies available nowadays was already implemented using cold atoms [5], ions [6], or photonics [7]. In particular, we mention a recent experiment [8] with coupled (linear) arrays of optical waveguides simulating a discrete version of SO coupling, which in the present Letter, is considered for the matter waves.

Meanwhile, the physics of BECs is characterized by two essential factors, which are not typical for the physics which is aimed to be simulated. First, a BEC is a nonlinear system, with the nonlinearity stemming from the two-body interactions [9]. Moreover, by changing the configuration of the system (i.e., of the laser beams) one can create effective nonlinear interactions of different types [10]. Respectively, such nonlinear objects as skyrmions [11], solitons $[12,13]$, antiferromagnetic structures, and symmetry breaking [14] have been recently reported for SO BECs. The second feature, is that a (quasi-) stationary state of the condensate requires the presence of the external potential. This, in particular, imposes constraints on the lower bound of the kinetic energy, what is particularly relevant for SO BECs (see, e.g., [14]). What concerns the external potentials, since the very first experiments with cold atoms loaded in optical lattices [15], it was widely recognized that lattices (i.e., periodic potentials) are particularly efficient in manipulating BECs [16]. Nowadays, the nonlinear properties of the atomic BECs held in optical lattices, described in the mean-field approximation are very well studied [17] (see also [18] for a brief review on BEC mixtures in optical lattices). It is, therefore, natural that studies of SO BECs in periodic potentials were already initiated. Manipulations with the band gap structure [19] and vortex lattice states [20] supported by SO coupling and optical lattices have being reported.

On the other hand, a few years ago there have been initiated studies of the properties of electronic gas with Rashba [21] and Dresselhaus [22] SO coupling in periodic structures. Those studies included the effect of dimensionality and features of the band gap structures of a gas in a superimposed periodic and arbitrary confining potentials [23], ballistic transport of electrons in periodic magnetic field [24,25], switching controlled by SO coupling [26], and quantum wire superlattices [27]. It was shown that SO coupling induced by an external periodic field leads to the appearance of new gaps, making the electronic system tunable even in the condensed matter settings.

Even more recently, the SO coupling was emulated in experiments with light propagating in arrays optical waveguides [8], thus opening a root towards new physical systems where the interplay of SO coupling and nonlinearity (if waveguides are of the Kerr type) can result in novel optical phenomena.

Bearing in mind the described findings as well as experimental availability of the spatially periodic Zeeman field [28], it is natural to address the effect of the lattice induced by such a field on the nonlinear properties of SO BECs. In this Letter, we, for the first time, perform thorough analysis of unusual properties, symmetries, and stability of gap solitons in spinor condensates with 
periodically coupled components. We show that the periodic magnetic fields applied to a SO BEC can support symmetric and asymmetric lattice solitons, classified with respect to their parity $(P)$, time $(T)$, and pseudocharge $(C)$ symmetries. The chemical potentials of the solitons originate from different gaps in the spectrum of the magnetic lattice for both attractive and repulsive interactions.

We address one-dimensional (1D) mixture of a twocomponent SO BEC which is described by a spinor $\boldsymbol{\Psi}=\operatorname{col}\left(\Psi^{(1)}, \Psi^{(2)}\right)$. In particular, for a tripod-type atomic scheme, like the one considered in [29], the components $\Psi^{(1)}$ and $\Psi^{(2)}$ are the wave functions of the two dark states emulating (pseudo)spins $|\uparrow\rangle$ and $|\downarrow\rangle$. Adopting the units where $\hbar=m=1$, and taking into account that the difference between spin-independent and spin-dependent twobody interactions can be made as small as necessary (in [1], the difference was of the order of $1 \%$ of the interaction magnitude), we explore the Hamiltonian of the system in the form $H=\int_{-\infty}^{\infty} \boldsymbol{\Psi}^{\dagger}\left(\mathcal{H}+(g / 2) \boldsymbol{\Psi}^{\dagger} \boldsymbol{\Psi}\right) \boldsymbol{\Psi} d x$, where the linear Hamiltonian $\mathcal{H}=-(1 / 2) \partial_{x}^{2}+(1 / 2) \Omega(x) \sigma_{3}+$ $i \kappa \sigma_{1} \partial_{x}$ accounts for the periodically modulated Zeeman field $\Omega(x)$ and for SO coupling with the strength $\kappa$ (which depends on the angle between optical beams and can be tuned [30]), $g=\operatorname{sign} a_{s}$ characterizes the strength of twobody interactions with the scattering length $a_{s}$, and $\sigma_{1,2,3}$ are the Pauli matrices. The nonlinear dynamics of the condensate is then governed by the coupled GrossPitaevskii equations (GPEs)

$i \boldsymbol{\Psi}_{t}=-\frac{1}{2} \boldsymbol{\Psi}_{x x}+\frac{\sigma_{3}}{2} \Omega(x) \boldsymbol{\Psi}+i \kappa \sigma_{1} \boldsymbol{\Psi}_{x}+g\left(\boldsymbol{\Psi}^{\dagger} \boldsymbol{\Psi}\right) \boldsymbol{\Psi}$.

We are interested in the stationary solutions having the form $\boldsymbol{\Psi}(x, t)=e^{-i \mu t} \boldsymbol{\psi}(x)$, where $\mu$ is a chemical potential and $\boldsymbol{\psi}(x)$ solves the stationary coupled GPEs. For the sake of definiteness, we consider a particular form of the Zeeman field

$$
\Omega(x)=2[\Delta+\delta \cos (2 x)],
$$

where $\Delta \geq 0$ and $\delta \geq 0$ are the average and the modulation amplitude of the field, respectively, and without loss of generality, the period is taken to be $\pi$. Nevertheless, we emphasize that the approach developed below is valid for more generic forms of Zeeman fields obeying the symmetries specified below.

First, we take into account that $\Omega(x)$ is an even function, $\Omega(x)=\Omega(-x)$, and obtain that if $\boldsymbol{\psi}(x)$ is a solution of Eq. (1) then the spinor $P T \boldsymbol{\psi}(x)=\overline{\boldsymbol{\psi}}(-x)$ (an overbar stands for the complex conjugation) defined by the parity and time inversions [31], is a solutions of the stationary GPEs, as well. At $\Delta=0$, the field given by Eq. (2) allows for additional symmetry reductions. The first one is readily seen in the shifted spatial variable $\tilde{x}=x+\pi / 4$. Indeed, since $\Omega(\tilde{x})=-\Omega(-\tilde{x})$, if $\boldsymbol{\psi}(\tilde{x})$ is a solution of Eq. (1) then the function obtained through the reduction $C P T \boldsymbol{\psi}(\tilde{x})=\sigma_{1} \overline{\boldsymbol{\psi}}(-\tilde{x})$, is also a solution ( $C$ by analogy with the charge symmetry [32] indicates the symmetry related to the inversion of the internal degree of freedom, which is the pseudospin in our case). Finally, due to the property $\Omega(x)=-\Omega(x+\pi / 2)$, one more symmetry stems from the possibility of flipping spin components in the $\pi / 2$ shifted lattice: $S \boldsymbol{\psi}(x)=\sigma_{1} \boldsymbol{\psi}(x+\pi / 2)$ is a solution of the stationary GPEs.

Bearing in mind the symmetries of the GPEs, it is natural to use them for classifying the solutions, i.e., to identify solutions which would be the eigenstates of the respective $P T, C P T$, or $S$ operators. It is important, however, that the $P T$ and $C P T$ symmetries imposed on the solutions rule out the phase invariance which is an attribute of the GPEs, because $e^{i \alpha}$ with a real $\alpha$ is not commutative with the $T$ operator. This artificial constraint on the solutions of the GPEs can be avoided by generalizing the definition of the symmetric modes. Namely, we define a $P T[C P T]$ symmetric solution, $\boldsymbol{\psi}^{P T}(x)\left[\boldsymbol{\psi}^{C P T}(x)\right]$, as solutions for which there exist a constant phase $\alpha$ transforming it into eigenfunctions of the respective symmetry operator, i.e., ensuring $e^{i \alpha} \boldsymbol{\psi}^{P T}(x)=P T\left(e^{i \alpha} \boldsymbol{\psi}^{P T}(x)\right)$ $\left[e^{i \alpha} \boldsymbol{\psi}^{C P T}(\tilde{x})=C P T\left(e^{i \alpha} \boldsymbol{\psi}^{C P T}(\tilde{x})\right)\right]$.

Now, we turn to a possibility of bifurcations of nonlinear modes obeying the above symmetries from the respective linear Bloch spinors $\varphi_{n k}(x)=\operatorname{col}\left(\varphi_{n k}^{(1)}(x), \varphi_{n k}^{(2)}(x)\right)$ which solve the spectral problem $\mathcal{H} \varphi_{n k}=\mu_{n}(k) \varphi_{n k}$, where $k$ refers to the wave vector in the reduced Brillouin zone (BZ), while $n$ indicates the number of the branch corresponding to the given $k$ with $n=1$ being the lowest one. The symmetries described above obviously hold also for the eigenfunctions of $\mathcal{H}$.

In Fig. 1, we show three typical band gap spectra $\mu_{n}(k)$. In the absence of the constant splitting $(\Delta=0)$ and at relatively weak SO coupling, the lower band edges are reached only at the boundary of the BZ [Fig. 1(a)]. Increase of the SO coupling results in closure of the first finite gap and appearance of the crossing points in the spectrum, as well as in a shift of the band gap edges to some internal points of the BZ [Fig. 1(b)] which are symmetric with respect to the center of the $\mathrm{BZ}$ and will be denoted as $\pm k_{0}$. Finally, in Fig. 1(c), we observe "reopening" of the first gap due to nonzero constant component of the Zeeman field $\Delta$. Notice that the lowest branches of the spectra in Figs. 1(b) and 1(c) resemble the well-known spectrum of the homogeneous SO BEC.

When the gap edge corresponds to the BZ boundary $k_{0}= \pm 1$ [Fig. 1(a)] due to the $P T$ symmetry, the Bloch spinor can be chosen as $\boldsymbol{\varphi}_{n 1}^{P T}(x)=\operatorname{col}\left(\boldsymbol{v}_{n}^{(1)}(x), i v_{n}^{(2)}(x)\right)$, where $v_{n}^{(1)}(x)=v_{n}^{(1)}(-x)$ and $v_{n}^{(2)}(x)=-v_{n}^{(2)}(-x)$ are real $2 \pi$-periodic functions. Such Bloch states give rise to $P T$-symmetric gap solitons: $\psi^{P T}(x)=P T \psi^{P T}(x)$, whose common property is that each spin component has symmetric distribution of the atomic density. Indeed, introducing the densities of the spinor components $n_{j}(x)=$ $\left|\psi^{(j)}(x)\right|^{2}$, one verifies that $n_{j}^{P T}(x)=n_{j}^{P T}(-x)$. For the 

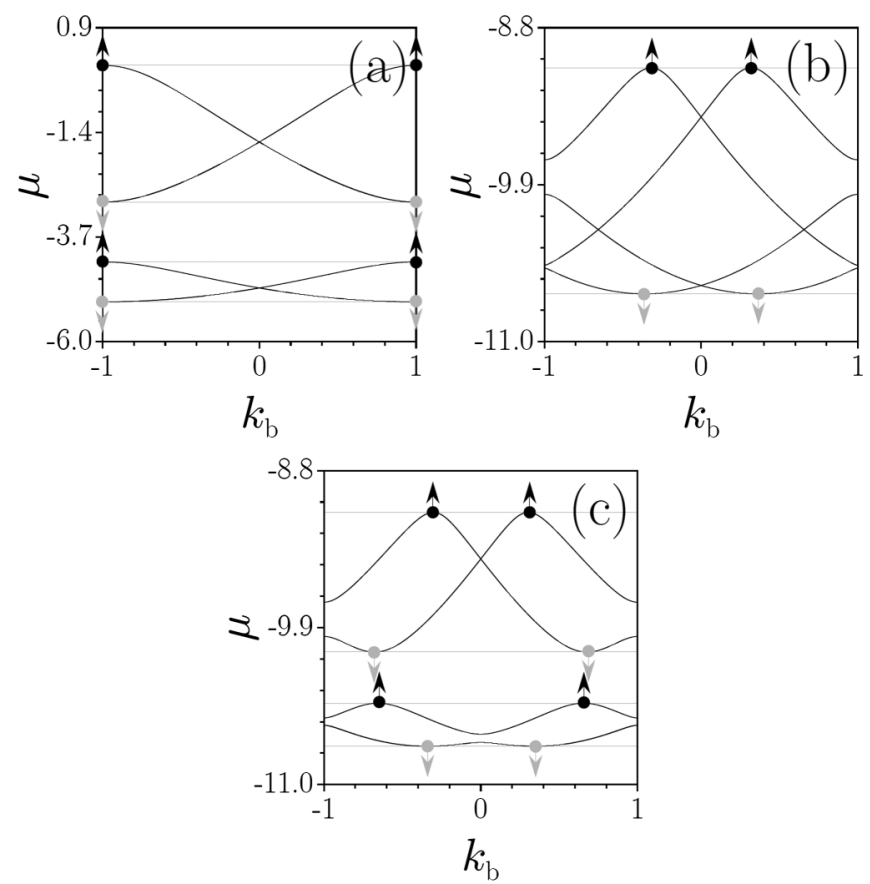

FIG. 1. The spectra of the Hamiltonian $\mathcal{H}$ with the periodic potential [Eq. (2)] for $\delta=6$ and (a) $\kappa=2, \Delta=0$ (b) $\kappa=4.5$, $\Delta=0$, (c) $\kappa=4.5, \Delta=1$. Horizontal gray lines indicate the band gap edges. Gray and black arrows indicate the points from which gap solitons bifurcate in the case of attractive and repulsive nonlinearities, respectively.

case of attractive interactions such soliton belonging to the semi-infinite band is illustrated in Fig. 2(a) [here and below, all soliton solutions of Eq. (1) were obtained numerically using the iterative Newton method]. Notice, that while this situation is seemingly similar to the one observed in the homogeneous medium [12], the effective periodic potential induced by the Zeeman field is inverted for the different components resulting in an immiscible antiferromagnetic phase (the atoms of each component are located in the minima of the respective periodic potentials). A counterpart of the described solitons can be also obtained in a finite gap in the case of repulsive interatomic interactions, see Fig. 2(b). Although there exists a general qualitative similarity with the soliton in the semi-infinite gap [cf. Fig. 2(a)], now the domains of the atomic concentration (i.e., the points where density is maximal), especially in the second component, do not coincide with the local minima of the effective potential for the respective component.

Turning to $C P T$-symmetric solitons, we observe that their characteristic feature is the equal number of atoms $N_{j}=\int_{-\infty}^{\infty} n_{j}(x) d x$ in the both components, $N_{1}=N_{2}$, since the densities are now related as: $n_{1}^{C P T}(\tilde{x})=n_{2}^{C P T}(-\tilde{x})$. Since in the spinor BEC the components are associated with the opposite spins $+(1 / 2)$ (the state $|\uparrow\rangle)$ and $-(1 / 2)$ (the state $|\uparrow\rangle$ ), one can introduce the (pseudo) magnetization density vectors $M_{1}=N_{1} / N$ and $M_{2}=-N_{2} / N$ of the
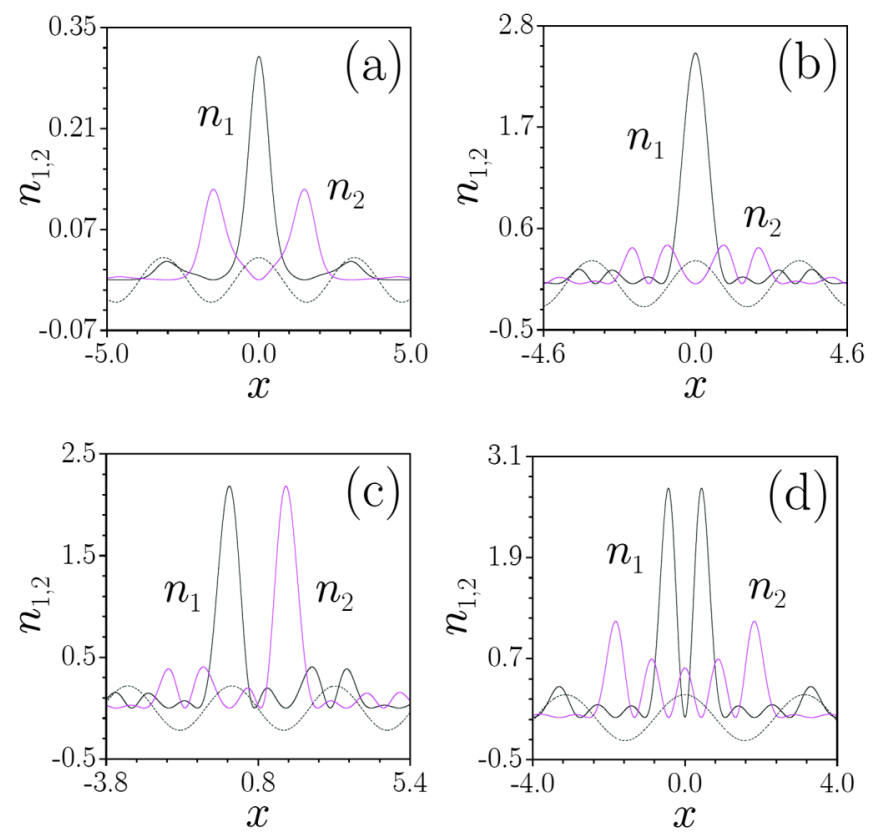

FIG. 2 (color online). Densities of a $P T$-symmetric soliton in the semi-infinite gap at $\mu=-5.23$ obtained for the attractive nonlinearity (a); $P T$-symmetric (b) and $C P T$-symmetric (c) solitons with $\mu=-3$ originating from the first finite gap for the repulsive nonlinearity, and $P T$-symmetric soliton with $\mu=-4.03$ from the first finite gap for attractive nonlinearity (d). In all cases, $\delta=6, \kappa=2, \Delta=0$. The dashed line shows the lattice profile for the $|\uparrow\rangle$ component.

components, such that the total pseudomagnetization density [33] is given by $M=M_{1}+M_{2}=\left(N_{1}-N_{2}\right) / N$. Using this definition, we conclude that $C P T$-symmetric solitons have zero total magnetization. An example of such a gap soliton emerging from the first finite gap in a repulsive condensate is presented in Fig. 2(c). In such modes, the maxima of density distributions in the two components are separated by a half of the lattice period and are located in neighboring extrema of the Zeeman lattice $\Omega(x)$.

Finally, in Fig. 2(d), we show a $P T$-symmetric soliton emerging from the first finite gap in the SO BEC with attractive interactions. Now the symmetries of the spinor components are "inverted" as compared to the case shown in Fig. 2(a), i.e., now $\psi^{(2)}(x)=\psi^{(2)}(-x)$ and $\psi^{(1)}(x)=$ $-\psi^{(1)}(-x)$. The reason for this "inversion" is that the soliton bifurcates from the upper gap edge [see the upper red arrow in Fig. 1(a)], thus resulting in the change of the sign of the effective mass.

The total numbers of atoms and magnetizations as functions of the chemical potential are shown in Fig. 3. For all solitons, $N$ demonstrates the typical behavior known for the one-component small-amplitude gap solitons [17]: near $n$th gap edge $N \sim \epsilon$, where $\epsilon=$ $\left(\left|\mu_{n}\left(k_{0}\right)-\mu\right| /|\mu|\right)^{1 / 2} \ll 1$. This indicates that the envelopes $A\left(\epsilon^{2} t, \epsilon x\right)$ of all bifurcating solitons in the small 

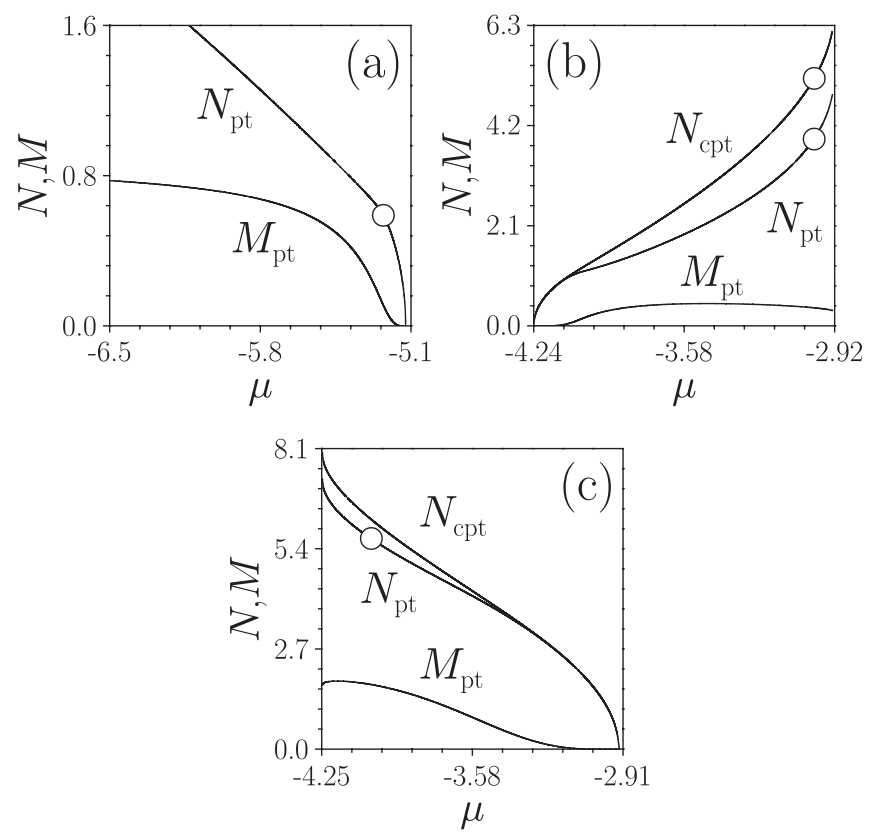

FIG. 3. The number of particles and magnetization vs $\mu$ for $P T$-symmetric solitons emerging from the semi-infinite gap in the case of attractive nonlinearity (a), for $P T$ and $C P T$ symmetric solitons emerging from the first finite gap in the case of repulsive (b) and attractive (c) nonlinearity. In (c), magnetization is multiplied by 10 . Circles correspond to solitons shown in Fig. 2. In all cases, $\delta=6, \kappa=2, \Delta=0$.

amplitude limit, $\boldsymbol{\psi}(x) \approx \epsilon A\left(\boldsymbol{\epsilon}^{2} t, \boldsymbol{\epsilon}\right) \boldsymbol{\varphi}_{n k_{0}}(x) e^{-i \mu_{n}\left(k_{0}\right) t}$, are described by the nonlinear Schrödinger (NLS) equation. In the panels $\mathrm{b}$ and $\mathrm{c}$, one observes that the branches of $P T$ and $C P T$ symmetric solitons seemingly merge. This, however, occurs only on the plane $(N, \mu)$ and do not represent a bifurcation. Both types of the solutions bifurcate from the different linear modes (obeying the respective symmetries). Coincidence of the branches close to the gap edge is explained by the $S$ symmetry ensuring the equality of the spatially averaged densities of the two components in the linear Bloch spinors, from which the respective solitons bifurcate. $C P$ and $C P T$ solitons in the first finite gap have also different stability [34]. In the case of repulsive interactions, $P T$ solitons are stable (unstable) at lower (larger) amplitudes. $C P T$ solitons are unstable, while in the vicinity of the bifurcation point, they can be classified as metastable ones as the instability increment is small enough $(<0.03)$ within the region of width $\left|\mu_{3}(1)-\mu\right| \approx 0.3$ adjacent to the upper gap edge located at $\mu_{3}(1) \approx-4.24$. In the condensate with negative scattering length, the $C P T$-symmetric solitons are always unstable while in the vicinity of the band edge, i.e., in the small-amplitude limit (with the width of the metastable domain $\left|\mu_{3}(1)-\mu\right| \approx$ 0.3 ), the $P T$-symmetric solitons can be metastable.

The magnetization shows rather complex behavior. For sufficiently small detuning $\epsilon$, one observes the behavior approximated by the law $M \sim \epsilon^{4}$ for all types of the
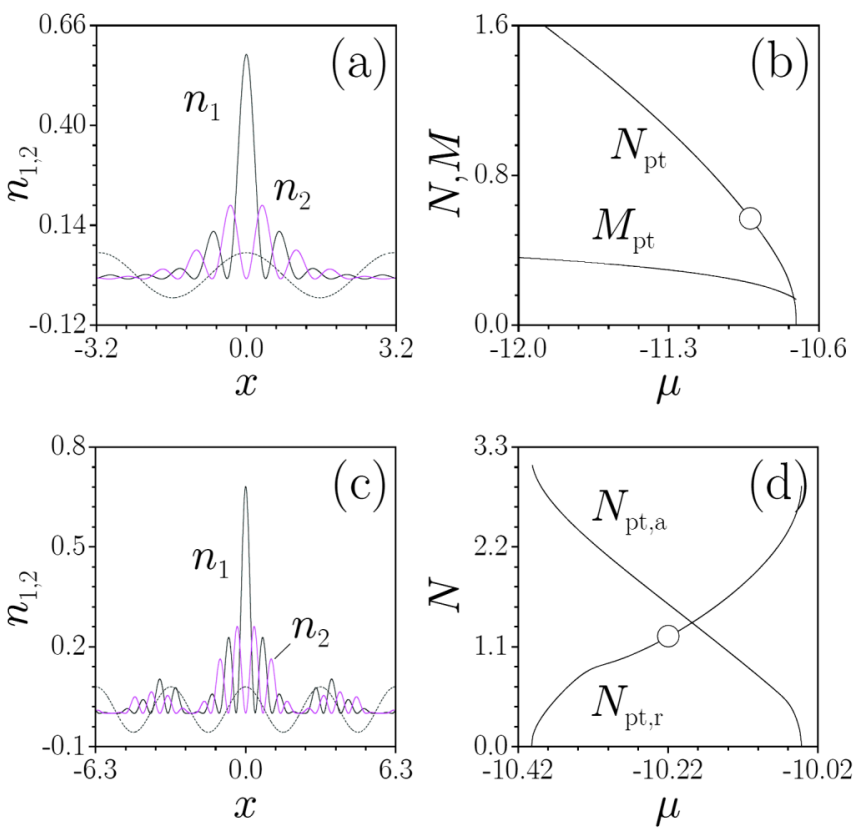

FIG. 4 (color online). (a) A gap-stripe soliton from the semiinfinite gap at $\mu=-10.92$ obtained for attractive nonlinearity. (b) The number of particles and magnetization vs $\mu$ in the attractive condensate. (c) A gap-stripe soliton from the first finite gap at $\mu=-10.22$ for repulsive nonlinearity corresponding to the circle in panel (d), which shows $N$ vs $\mu$ for repulsive and attractive interactions in the first finite gap. In all cases, $\delta=6$, $\kappa=4.5, \Delta=1$.

reported $P T$-symmetric solutions [recall that for the $C P T$-solitons, like the one shown in Fig. 2(c), the total magnetization is zero]. As above, this is explained by the symmetry $S$, ensuring that at small amplitudes, the states $|\uparrow\rangle$ and $|\downarrow\rangle$ are equally populated. At large detuning, the magnetization may start to decay [see Fig. 3(b)].

Now we turn to the situation where a band gap is achieved in some internal points $\pm k_{0}$ corresponding to a double degenerate eigenvalue $\mu_{n}^{(0)}=\mu_{n}\left( \pm k_{0}\right)$ [see Figs. 1(b) and 1(c)]. In the homogeneous case, a similar situation gives origin to a stripe phase [35] in repulsive condensate or to a stripe soliton [12] in a condensate with a negative scattering length. In order to construct a $P T$-symmetric solution, we depart from one of the superposition of the linear spinors, say $\boldsymbol{\varphi}_{0}(x)=\boldsymbol{\varphi}_{n k_{0}}(x)+$ $\boldsymbol{\varphi}_{n,-k_{0}}(x)$, where the relation between the Bloch states is given by $\boldsymbol{\varphi}_{n,-k_{0}}(x)=\sigma_{3} \overline{\boldsymbol{\varphi}}_{n k_{0}}(x) . P T$-symmetric gap-stripe solitons belonging to the branch bifurcating from $\varphi_{0}(x)$ are illustrated in Fig. 4 for the lattice whose band gap structure is presented in Fig. 1(c). Gap-stripe soliton in the semiinfinite gap (upper row of Fig. 4) are stable in the whole domain of the existence. In the finite gap, the solitons are generically unstable for the depth of potential considered here, although we have observed (numerically) stable time evolutions of the gap-stripe solitons in the case of repulsive interactions which was accompanied by regular 
oscillations of the amplitude, indicating on a possibility of a breather solution. Comparing the gap-stripe solitons with the "conventional" gap solitons considered above, we find that while the envelope of stripe solitons is also described by the NLS equation, i.e., $N \sim \epsilon$ close to the gap edge, the total magnetization now displays very different behavior near the bifurcation point: it approaches a nonzero value. This difference with the cases of the $P T$-symmetric solitons follows from the structure of the linear Bloch spinors, which now do not obey S symmetry and thus, the states $|\uparrow\rangle$ and $|\downarrow\rangle$ have different populations. In other words, such states have nonzero magnetization even in the linear limit.

To conclude, we have found a diversity of stable gap and gap-stripe solitons in spin-orbit-coupled Bose-Einstein condensate subject to a spatially periodic Zeeman field. The obtained solutions can be classified by the major physical symmetries, as being invariant with respect to either $P T$ or $C P T$ reductions. The respective solitons have either nonzero or zero total magnetization. The conventional gap and gap-stripe solitons are observed in lattices with different parameters, while solitons of the same type but obeying different symmetries can be observed in the same lattice but at different spatial locations. All the obtained solutions obey an antiferromagnetic structure and thus, represent immiscible phases.

V. V. K. acknowledges support of the FCT (Portugal) Grant No. PEst-OE/FIS/UI0618/2011. F. K. A. acknowledges support from the Fundação Amparo e Pesquisa de São Paulo (FAPESP, Brasil).

[1] Y. J. Lin, K. Jimenez-Garcia, and I. B. Spielman, Nature (London) 471, 83 (2011).

[2] Y.-J. Lin, R. L. Compton, A. R. Perry, W. D. Phillips, J. V. Porto, and I. B. Spielman, Phys. Rev. Lett. 102, 130401 (2009); Y.-J. Lin, R. L. Compton, K. Jimenez-Garca, J. V. Porto, and I. B. Spielman, Nature (London) 462, 628 (2009); C. H. L. Quay, T. L. Hughes, J. A. Sulpizio, L. N. Pfeiffer, K.W. Baldwin, K. W. West, D. GoldhaberGordon, and R. de Picciotto, Nat. Phys. 6, 336 (2010).

[3] V. Galitski and I. B. Spielman, Nature (London) 494, 49 (2013).

[4] P. Hauke, F. M. Cucchietti, L. Tagliacozzo, I. Deutsch, and M. Lewenstein, Rep. Prog. Phys. 75, 082401 (2012).

[5] I. Bloch, J. Dalibard, and S. Nascimbène, Nat. Phys. 8, 267 (2012).

[6] R. Blatt and C.F. Roos, Nat. Phys. 8, 277 (2012).

[7] A. Aspuru-Guzik and P. Walther, Nat. Phys. 8, 285 (2012).

[8] R. Keil, J. M. Zeuner, F. Dreisow, M. Heinrich, A. Tünermann, S. Nolte, and A. Szameit, Nat. Commun. 4, 1558 (2013).

[9] C. Pethick and H. Smith, Bose-Einstein Condensation in Dilute Gases (Cambridge University Press, Cambridge, 2002); L.P. Pitaevskii and S. Stringari, Bose-Einstein Condensation (Clarendon Press, Oxford and New York, 2003).
[10] Y. Zhang, L. Mao, and C. Zhang, Phys. Rev. Lett. 108, 035302 (2012).

[11] T. Kawakami, T. Mizushima, M. Nitta, and K. Machida, Phys. Rev. Lett. 109, 015301 (2012).

[12] V. Achilleos, D. J. Frantzeskakis, P. G. Kevrekidis, and D. E. Pelinovsky, Phys. Rev. Lett. 110, 264101 (2013).

[13] Y. Xu, Y.Zhang, and B. Wu, Phys. Rev. A 87, 013614 (2013).

[14] D. A. Zezyulin, R. Driben, V. V. Konotop, and B. A. Malomed, Phys. Rev. A 88, 013607 (2013).

[15] B. P. Anderson and M. A. Kasevich, Science 282, 1686 (1998).

[16] O. Morsch and M. Oberthaler, Rev. Mod. Phys. 78, 179 (2006).

[17] V. A. Braznyi and V. V. Konotop, Mod. Phys. Lett. B 18, 627 (2004); R. Carretero-González, D. J. Frantzeskakis, and P. G. Kevrekidis, Nonlinearity 21, R139 (2008).

[18] H. A. Cruz, V. A. Brazhnyi, V. V. Konotop, and M. Salerno, Physica (Amsterdam) 238D, 1372 (2009) and references therein.

[19] Y. Zhang and C. Zhang, Phys. Rev. A 87, 023611 (2013).

[20] H. Sakaguchi and B. Li, Phys. Rev. A 87, 015602 (2013).

[21] Y. A. Bychkov and E. I. Rashba, J. Phys. C 17, 6039 (1984).

[22] G. Dresselhaus, Phys. Rev. 100, 580 (1955).

[23] S. Smirnov, D. Bercioux, and M. Grifoni, Europhys. Lett. 80, 27003 (2007); V. Ya. Demikhovski, D. V. Khomitsky, and A. A. Perov, J. Low Temp. Phys. 33, 115 (2007).

[24] J.-F. Liu, W.-J. Deng, K. Xia, C. Zhang, and Z. Ma, Phys. Rev. B 73, 155309 (2006).

[25] X.B. Xiao, X.M. Li, and Y.G. Chen, Physica (Amsterdam) 404B, 4159 (2009).

[26] S. J. Gong and Z. Q. Yang, J. Phys. Condens. Matter 19, 446209 (2007).

[27] G. Thorgilsson, J.C. Egues, D. Loss, and S. I. Erlingsson, Phys. Rev. B 85, 045306 (2012).

[28] K. Jiménez-García, L. J. LeBlanc, R. A. Williams, M. C. Beeler, A. R. Perry, and I. B. Spielman, Phys. Rev. Lett. 108, 225303 (2012).

[29] M. J. Edmonds, J. Otterbach, R. G. Unanyan, M. Fleischhauer, M. Titov, and P. Öhberg, New J. Phys. 14, 073056 (2012).

[30] J. Dalibard, F. Gerbier, G. Juzeliünas, and P. Öhberg, Rev. Mod. Phys. 83, 1523 (2011).

[31] The Wigner time inversion operator $T \mathbf{\Psi}(t, x)=\overline{\mathbf{\Psi}}(-t, x)$ applied to a stationary mode $\boldsymbol{\Psi}(t, x)=e^{i \mu t} \boldsymbol{\psi}(x)$ is reduced to the complex conjugation.

[32] V.B. Berestetskii, L.P. Pitaevskii, and E. M. Lifshitz, Quantum Electrodynamics (Elsevier, Oxford, 1982).

[33] see, e.g., L. D. Landau, E. M. Lifshitz, and L. P. Pitaevskii, Electrodynamics of Continuous Media (Elsevier, Oxford, 2004).

[34] In the present Letter, we have performed the linear stability analysis, whose details are presented in the Supplemental Material at http://link.aps.org/ supplemental/10.1103/PhysRevLett.111.060402. The stability of soliton solutions, including ones shown in the figures, was independently checked by the direct numerical integration of Eq. (1) with weakly perturbed soliton inputs.

[35] T.L. Ho and S. Zhang, Phys. Rev. Lett. 107, 150403 (2011); S. Sinha, R. Nath, and L. Santos, Phys. Rev. Lett. 107, 270401 (2011); Y. Li, L. P. Pitaevskii, and S. Stringari, Phys. Rev. Lett. 108, 225301 (2012). 